\title{
Evaluation of Head and Neck Position on Oropharyngeal Leak Pressure with Baska Mask and Streamlined Liner of Pharyngeal Airway (SLIPA ${ }^{\mathrm{TM}}$ ): Arandomized Clinical Trial
}

\author{
Rehab Abdel-Raof Abdel-Aziz ${ }^{*}$, Yasser Mohamed Osman \\ Department of Anesthesia and Surgical Intensive Care, Faculty of Medicine, Alexandria University, Alexandria, Egypt
}

Email address:

trcium2002@yahoo.com (R. Abdel-Raof Abdel-Aziz), yasseralx@hotmail.com (Y.M. Osman)

${ }^{*}$ Corresponding author

\section{To cite this article:}

Rehab Abdel-Raof Abdel-Aziz, Yasser Mohamed Osman. Evaluation of Head and Neck Position on Oropharyngeal Leak Pressure with Baska Mask and Streamlined Liner of Pharyngeal Airway (SLIPA ${ }^{\mathrm{TM}}$ ): Arandomized Clinical Trial. Journal of Anesthesiology.

Vol. 5, No. 5, 2017, pp. 36-41. doi: 10.11648/j.ja.20170505.11

Received: November 2, 2017; Accepted: November 16, 2017; Published: December 25, 2017

\begin{abstract}
The primary objective was to compare oropharyngeal leak pressures of streamlined liner of pharyngeal airway $\left(\mathrm{SLIPA}^{\mathrm{TM}}\right)$ and Baska mask at different head and neck positions. Assessing the fiberoptic view of glottis and ventilation score of both devices in different positions were the secondary objectives. Sixty patients ASA I-II, 18-60 years of either sex scheduled for short ambulatory surgery were included. Patients were randomly assigned in a 1:1 ratio to either the SLIPA ${ }^{\mathrm{TM}}$ or $^{2}$ the Baska mask group. The effect of various head and neck positions was evaluated. Neutral position was maintained first then the patient was repositioned in the following positions: maximal extension, maximal flexion, and maximal rotation to the left. In each position, peak airway pressure and oropharyngeal leak pressure were noted. The ventilation score was assessed and fibreoptic views were noted. In both groups, oropharyngeal leak pressures and peak airway pressure were significantly higher with flexion. The fiberoptic score frequently decreased in flexion but with no effect on the ventilation. In comparison between the two devices, there was no significant change in the fibreoptic view of the glottis at different neck positions. So we concluded that, effective ventilation is possible with both Baska mask and SLIPA ${ }^{\mathrm{TM}}$ with the head in neutral, flexion, extension, and lateral rotation positions. But care should be taken with extreme flexion and the airway pressures need to be monitored. Baska mask has a better margin of safety than SLIPA ${ }^{\mathrm{TM}}$ due to better airway sealing pressures.
\end{abstract}

Keywords: Head and Neck Position, Oro-Pharyngeal Leak Pressure, Airway Devices

\section{Introduction}

Supraglottic airway devices (SGA) are used extensively during general anesthesia instead of tracheal intubation because of its less invasive nature [1]. The streamlined liner of the pharynx airway $\left(\mathrm{SLIPA}^{\mathrm{TM}}\right)$ and the Baska mask are two of the latest addition to the Supraglottic airway devices.

The streamlined liner of the pharynx airway $\left(\mathrm{SLIPA}^{\mathrm{TM}}\right)$ is a non-cuffed single-use SGA, its shape is designed to stick to the pharynx and palate $[2,3]$. The shape of SLIPA ${ }^{\mathrm{TM}}$ has a hollow body which allows it to fit in the patient's pharynx, sealing it without the use of an inflatable cuff. Its unique shape allows it to maintain a secure airway during positive pressure ventilation without the need to use any kind of fixation straps. Also it has a 50-ml empty internal space which allows the removal of pharyngeal secretions, thus reducing the risk of pulmonary aspiration $[2,4]$. The Baska mask is a new single use SGA which is designed by Australian anesthetists Kanag and Meena Baska [5, 6]. The Baska mask has no orogastric tube but instead of it a sump and two drains are present [7]. Also its whole length of the airway tube is fortified with a bite block and is oval in shape. That unique oval shape fits the shape of the mouth and reduces rotation within the pharynx. Head and neck movement can lead to changes in the shape of the pharynx. This shape changes can alter the forces transmitted along the airway device during ventilation which may lead to displacement of the SGA and increasing the airway leak [8]. 
The primary objective of this study is to determine the effect of different head and neck positions namely, neutral, flexion, extension, and left rotation on the oropharyngeal leak pressures when SLIPA ${ }^{\mathrm{TM}}$ and the Baska mask are used. The secondary objectives are to assess and compare the fiberoptic view of the glottis, ventilation score, of both devices in different head and neck positions.

\section{Methods}

\subsection{Patients and Study Design}

After approval from the Institutional Ethics Committee of the Alexandria Main University Hospitals, this study was conducted on sixty patients of either sex with ASA physical status I-II. Their age ranged between 18-60 years and scheduled for short duration $(<3 \mathrm{hr})$ ambulatory surgery. A written consent was obtained from all of the patients participating in the study after explaining the procedure of the study in details. The study was registered at the Pan African Clinical Trials Registry under the number PACTR201701001985265.

Patients with body mass index $\geq 25 \mathrm{~kg} / \mathrm{m}^{2}$ or have history of obstructive sleep apnea, or history of gastro-esophageal reflux were excluded from the study. Also patients with high risk of aspiration, anticipated difficult airway, any pathology of the neck, upper respiratory tract infection or potentially have full stomach were excluded. Patients were randomly divided into two equal groups either the SLIPA ${ }^{\mathrm{TM}}$ or the Baska mask group (30 patients in each group). Randomization was done using the computer and the result was held in sealed envelopes until the time of the operation. Preoperative examination of the maximum neck extension, flexion, and rotation were performed to all patients.

\subsection{Surgical Procedure and Clinical Observations}

On arrival to the operating room, standard monitoring (pulse oximetry, non-invasive blood pressure recording, electrocardiography, and capnography) was connected to the patient. An Intravenous line was inserted. Then a base line reading of blood pressure (BP), heart rate (HR), and peripheral oxygen saturation $\left(\mathrm{SpO}_{2}\right)$ were taken. Preoxygenation with $100 \%$ oxygen for three minutes, then intravenous induction of anesthesia was done using fentanyl $2 \mu \mathrm{g} / \mathrm{kg}$, propofol $2.5 \mathrm{mg} / \mathrm{kg}$ and $0.5 \mathrm{mg} / \mathrm{kg}$ atracurium besylate. Mask ventilation was done for four minutes with $4 \%$ sevoflurane in $100 \%$ oxygen till full muscle relaxation was obtained. The patient head is put in the sniffing position. An experienced anesthesiologist inserted the lubricated supraglottic airway device chosen for that patient [7, 8]. Size selection of the SLIPA ${ }^{\mathrm{TM}}$ and Baska mask was based on manufacturer guidelines.

For SLIPA $^{\mathrm{TM}}$ group, for males less than $165 \mathrm{~cm}$ in height size 51 was used, while size 53 for those between 165 and $175 \mathrm{~cm}$, and size 55 for those over $175 \mathrm{~cm}$. on the other hand size 47 was used for female patients under $155 \mathrm{~cm}$ and size 49 if their height is between 155 and $165 \mathrm{~cm}$, and size 51 for those over $165 \mathrm{~cm}$. After opening the patient's jaw with one hand, the other hand was used to push the SLIPA ${ }^{\mathrm{TM}}$ into position so that the heel of the SLIPA $^{\mathrm{TM}}$ was in the nasopharynx over the base of the tongue.

In the Baska Mask group the sizes of the device is chosen according to patient weight: size three for patients weighing between ( 30 to $50 \mathrm{~kg}$ ), size four ( 50 to $70 \mathrm{~kg}$ ), size five (70 to $100 \mathrm{~kg}$ ), and size six for patient over $100 \mathrm{~kg}$. For its insertion, the firmer part of the mask was compressed by the fingers and pushed towards the hard palate till resistance was encountered.

After connecting the breathing circuit to the SLIPA ${ }^{\mathrm{TM}}$ or Baska mask, appropriate placement and ventilation were confirmed by observing the chest wall movement, auscultation of breath sounds and square-wave capnograph. In case of inadequate ventilation or failure to confirm the successful placement of the device the case would be removed from the study. The fresh gas flow was set at 4 $\mathrm{L} / \mathrm{min}$. Maintenance of anesthesia was continued with $2-3 \%$ sevoflurane in $40 \%$ oxygen. Incremental dose of $0.1 \mathrm{mg} / \mathrm{kg}$ atracurium was given when needed and tidal volume and the respiratory rate were adjusted to keep the end-tidal carbon dioxide $\left(\mathrm{EtCO}_{2}\right)$ between 35 and $40 \mathrm{~mm} \mathrm{Hg}$.

The ventilation score was calculated based on three criteria: the presence or absence of a leakage with an airway pressure of $15 \mathrm{cmH}_{2} \mathrm{O}$, bilateral chest excursion with a peak inspiratory pressure of $20 \mathrm{~cm} \mathrm{H}_{2} \mathrm{O}$ and a square wave capnogram, each item was given 0 if its item is absent or 1 point if it is present. Thus, the ventilation score equals 3 if all three criteria exist which is the maximum score $[9,10]$. To evaluate the effect of changing the position of the head and neck on the SGA the patient's head was put first in the neutral position in which a line joining the superior orbital margin and the external ear canal with the top of the shoulder is vertical. After that the patient's head was rotated to its maximum to the left then maximally extended and maximally flexed. Peak airway sealing pressure and oropharyngeal leak pressure were measured in each position at fixed tidal volume of $10 \mathrm{~mL} / \mathrm{kg}$ using a volume-controlled ventilator. All the data were collected three minutes after each position adjustment.

Oropharyngeal leak pressure was measured by putting the patient on a bag or manual mode with a closed adjustable pressure limiting (APL) valve and a fixed gas flow of $3 \mathrm{~L} / \mathrm{min}$ $[11,12]$ Airway pressure was allowed to increase (but not to exceed $40 \mathrm{~cm} \mathrm{H}_{2} \mathrm{O}$ ) until a leakage sound is heard through a stethoscope placed over the patient's mouth. If no sound was heard until $40 \mathrm{~cm} \mathrm{H}_{2} \mathrm{O}$ then the OLP was recorded as $>40 \mathrm{~cm}$ $\mathrm{H}_{2} \mathrm{O}$ [12].

An independent anesthetist with no knowledge of the study and is highly experienced in using the fibreoplic scope commented on the fibreoptic views using Brimacombe score. After passing a fibreoptic scope through the airway tube the tip of the fibreoptic was position $1 \mathrm{~cm}$ proximal to the end of the tube. Then the Brimacombe score was noted ( 1 if the vocal cords not seen, 2 if the vocal cords plus anterior epiglottis seen, 3 if the vocal cords plus posterior epiglottis 
seen, and 4 if only vocal cords visible) $[13,14]$. At the end of surgery, neuromuscular blockade was antagonized with 0.05 $\mathrm{mg} / \mathrm{kg}$ neostigmine and atropine $1 \mathrm{mg}$. The SAD was routinely removed after the subject had regained consciousness and adequately responded to verbal command.

\subsection{Statistical Analysis}

Sample size was calculated, with a confidence interval of $95 \%$ and a power of $90 \%$, based upon previous studies [10, 15]. The maximum sample size was 30 in each group for flexion with a difference between means of $4 \mathrm{~cm}$ of $\mathrm{H}_{2} \mathrm{O}$ for oro-pharyngeal leak pressures. SPSS Version 20 was used for statistical analysis. Statistical analysis was done with the paired $t$-test and unpaired $t$-test. Brimacombe scores and ventilation scores were compared using the Mann-Whitney test between groups and Wilcoxon test within the groups. $P<$ 0.05 was considered significant.

\section{Results}

Descriptive details of patients are shown in (Table 1), as 60 patients were included ( 27 men and 33 women); age and
BMI were 19-59 years, and 19-30 kg.m², respectively. Both groups were comparable as regard to demographic characteristics.

Table 1. Demographic data of both groups.

\begin{tabular}{llll}
\hline & SLIPA $^{\text {TM }}$ group & BM group & Pvalue \\
\hline Age & $19-59(39.9) \pm 11.1$ & $19-57(37.5) \pm 11.4$ & 0.424 \\
Sex M-F & $14-16$ & $13-17$ & \\
BMI & $19-30(25.8) \pm 3.12$ & $21-30(26.1) \pm 2.93$ & 0.641 \\
ASAI-II & $8-22$ & $7-23$ & \\
\hline
\end{tabular}

In the SLIPA ${ }^{\mathrm{TM}}$ group, compared with neutral position, oropharyngeal leak pressures were significantly higher with flexion and lower with extension but similar with rotation of head and neck. In Baska mask group, compared with neutral position, oropharyngeal leak pressures were significantly higher with flexion and lower with extension, but it was slightly higher with lateral rotation but without any statistical difference from the neutral position. There were statically difference between SLEPA $^{\mathrm{TM}}$ group and Baska group as regard oropharyngeal leak pressure with different position of head and neck (Table 2).

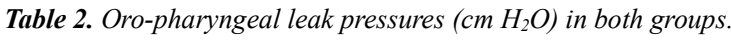

\begin{tabular}{lllll}
\hline \multirow{2}{*}{ Parameter } & SLIPA $^{\text {TM }}$ & & BASKA & Pvalue $^{\mathbf{2}}$ \\
\cline { 2 - 5 } & & Pvalue $^{\mathbf{1}}$ & & Pvalue $^{\mathbf{1}}$ \\
\hline Neutral & $23 \pm 3.7$ & & $27.3 \pm 3.77$ & $<0.001^{*}$ \\
Flexion & $24.8 \pm 4.12$ & $0.028345^{*}$ & $30 \pm 4.2$ & $0.001^{*}$ \\
Extension & $19.5 \pm 3$ & $0.000496^{*}$ & $22.46 \pm 4.2$ & $<0.001^{*}$ \\
Lateral rotation & $23.46 \pm 3.4$ & 0.293433 & $28.56 \pm 4.88$ & $0.003^{*}$ \\
\hline
\end{tabular}

Pvalue $^{1}$ is the $P$ value of different positions in comparison with neutral position in the same group.

Pvalue $^{2}$ is the $P$ value of different positions in comparison to the other group.

$P<0.05$ is considered significant.

In SLIPA $^{\mathrm{TM}}$ group, peak airway pressures were significantly higher with flexion and lower with extension but with no statistical significance, but with rotation of head and neck peak air way pressure significantly slightly increase in comparison with neutral position. (Table 3). In Baska group, peak airway pressures were significantly higher with flexion with statistical difference but it did not change significantly with extension or with rotation of head and neck. There were statically difference between SLEPA ${ }^{\mathrm{TM}}$ group and Baska group as regard peak airway pressure in neutral and flexion positions and with lateral rotation of the head, but without any significant difference during extension position (Table 3).

Table 3. Peak airway pressures $\left(\mathrm{cm} \mathrm{H}_{2} \mathrm{O}\right)$ in both groups.

\begin{tabular}{lllll}
\hline \multirow{2}{*}{ Parameter } & SLIPA $^{\text {TM }}$ & & BASKA & Pvalue $^{2}$ \\
\cline { 2 - 5 } & & Pvalue $^{\mathbf{1}}$ & Pvalue $^{\mathbf{1}^{\mathbf{2}}}$ \\
\hline Neutral & $14.2 \pm 2.83$ & & $16.7 \pm 3.8$ & $0.004^{*}$ \\
Flexion & $16.73 \pm 3.22$ & $0.001^{*}$ & $19.33 \pm 4.9$ & $0.014^{*}$ \\
Extension & $14.76 \pm 2.68$ & 0.21 & $15.3 \pm 3.39$ & $0.019^{*}$ \\
Lateral rotation & $15.6 \pm 3.02$ & $0.034^{*}$ & $17.46 \pm 3.9$ & 0.501 \\
\hline
\end{tabular}

Pvalue $^{1}$ is the $P$ value of different positions in comparison with neutral position in the same group.

Pvalue $^{2}$ is the $P$ value of different positions in comparison to the other group.

$P<0.05$ is considered significant.

Table 4. Ventilation score in both groups.

\begin{tabular}{lllll}
\hline $\begin{array}{l}\text { Ventilations } \\
\text { core(3/2/1/0) }\end{array}$ & Neutral & Flexion & Extension & $\begin{array}{l}\text { Lateral } \\
\text { rotation }\end{array}$ \\
\hline SLIPA $^{\mathrm{TM}}$ & $30 / 0 / 0 / 0$ & $29 / 1 / 0 / 0$ & $30 / 0 / 0 / 0$ & $30 / 0 / 0 / 0$ \\
BASKAMASK & $30 / 0 / 0 / 0$ & $30 / 0 / 0 / 0$ & $29 / 1 / 0 / 0$ & $29 / 1 / 0 / 0$ \\
\hline
\end{tabular}

The fiberoptic score frequently decreased in flexion (Tables 5, 6, and 7) but with no effect on the ventilation as shown in (Table 4). In comparison between the two devices, there was no significant change in the fibreoptic view of the glottis at different neck positions. 
Table 5. Fibreoptic view of glottis with SLIPA ${ }^{T M}$.

\begin{tabular}{lllll}
\hline Brimacombe score & Neutral & Flexion & Extension & $\begin{array}{l}\text { Lateral } \\
\text { rotation }\end{array}$ \\
\hline 4 & 12 & 9 & 13 & 9 \\
3 & 16 & 10 & 13 & 15 \\
2 & 1 & 7 & 3 & 5 \\
1 & 1 & 4 & 1 & 1 \\
$P$ value & & 0.361 & 0.072 & 0.240 \\
\hline
\end{tabular}

Data are in actual numbers. $P<0.05$ is considered significant.
Table 6. Fibreoptic view of glottis with Baska mask.

\begin{tabular}{lllll}
\hline Brimacombe score & Neutral & Flexion & Extension & $\begin{array}{l}\text { Lateral } \\
\text { rotation }\end{array}$ \\
\hline 4 & 14 & 13 & 14 & 11 \\
3 & 12 & 11 & 11 & 13 \\
2 & 2 & 2 & 3 & 5 \\
1 & 2 & 4 & 2 & 1 \\
$P$ value & & 0.062 & 0.091 & 0.141 \\
\hline
\end{tabular}

$\mathrm{P}$ value is the $P$ value of different positions in comparison with neutral position in the same group.

Table 7. Comparison between two groups as regard fibreoptic view of glottis (4/3/2/1).

\begin{tabular}{llllll}
\hline \multirow{2}{*}{ Parameter } & SLIPA & & BASKA & \\
\cline { 2 - 5 } & & P value $^{\mathbf{1}}$ & & \\
\hline Neutral & $12,16,1,1$ & & $14,12,2,2$ & \\
Flexion & $9,10,7,4$ & 0.360 & $13,11,2,4$ & 0.811 \\
Extension & $13,13,3,1$ & 0.072 & $14,11,3,2$ & 0.062 \\
Lateral rotation & $9,15,5,1$ & 0.240 & $11,13,5,1$ & 0.091 & 0.141 \\
\hline
\end{tabular}

Data are in actual numbers.

$\mathrm{P}_{\text {value }}{ }^{1}$ is the $\mathrm{P}$ value of different positions in comparison with neutral position in the same group.

Pvalue $^{2}$ is the $\mathrm{P}$ value of different positions in comparison to the other group.

$\mathrm{P}<0.05$ is considered significant.

\section{Discussion}

The main objective of this study was to compare theoropharyngeal leak pressures of SLIPA ${ }^{\mathrm{TM}}$ and Baska mask at different head and neck positions to find out which device is more suitable to be used when it is anticipated that the head position will not be in the neutral position during the surgery.

The pressure at which oropharyngeal leak happens when using positive pressure ventilation correlates to the degree of airway protection provided by the supra-glottic airway device used. This study demonstrated that the oro-pharyngeal leak pressure was significantly higher with Baska mask in comparison to SLIPA ${ }^{\mathrm{TM}}$ in all the head and neck positions tested (Table 2), indicating that Baska mask provides a safer seal for positive ventilation even in different head positions.

We showed that effective ventilation can be obtained with the head and neck extended, rotated or flexed using either Baska mask or SLIPA ${ }^{\mathrm{TM}}$ when we compared the ventilation score in those positions. These findings have significant implications for surgeries that require various head and neck positions.

Previous studies were done to assess the influence of head and neck position on ventilation with first and second generations of supraglottic air way devices such as I-gel, laryngeal tube suction (LTS), ProSeal laryngeal mask airway (PLMA) and Cobra perilaryngeal airway (CobraPLA). One of the earlier studies was done by Xue and Mao et al. [16] in which they evaluated the influences of head flexion on airway sealing pressure and quality of ventilation, through the ProSealTM laryngeal mask in 80 anesthetized adult patients. When compared to the neutral head position, the head flexed significantly improved the airway seal pressure and the quality of ventilation of the ProSeal LMA $(\mathrm{p}<0.05)$ and they concluded that head flexion improved airway seal and ventilation quality of the ProSeal LMA. A similar conclusion was demonstrated in this study showing the same effect of head flexion on ventilation and airway sealing when using Baska mask or SLIPA ${ }^{\mathrm{TM}}$.

Park and Han et al. [15] compared the oropharyngeal leak pressure in four head and neck positions: neutral, $45^{\circ}$ of flexion, $45^{\circ}$ of extension, and $45^{\circ}$ of right rotation and also difficulty in ventilation was assessed in their work, in which one-hundred-thirty-nine patients (aged 18-70 yr) scheduled for minor surgical procedures were randomly used one of three supraglottic airway devices; the laryngeal tube suction (LTS), ProSeal laryngeal mask airway (PLMA) and Cobra perilaryngeal airway (CobraPLA). They found that, the leak pressures of the PLMA were lowest in the extension (18.5 vs 23.9 and $26.8 \mathrm{~cm} \mathrm{H}_{2} \mathrm{O}$ of LTS and CobraPLA, respectively; $P$ $\leq 0.001)$ and in the rotation position $(25.0$ vs 29.4 and 28.5 $\mathrm{cm} \mathrm{H}_{2} \mathrm{O}$ of LTS and CobraPLA, respectively; $P \leq 0.005$ ). Also they found difficult in the ventilation in seven patients with LTS after neck flexion, which required tracheal intubation. They concluded that the PLMA showed significantly lower oropharyngeal leak pressures than did the LTS or CobraPLA in the neck extension and rotation positions and should take care when changing the position of the head and neck when using the Cobra-PLA or LTS as ventilatory difficulty may occur. In contrast using the Baska mask or the SLIPA ${ }^{\mathrm{TM}}$ did not show any significant ventilation impairment in any head position.

Sanuki et al. [10] started the evaluation of effectiveness of head and neck positions on ventilation on I-gel airway devices, as they investigated the effect of different positions on oropharyngeal leak pressure and ventilation scores during ventilation with I-gel. Compared with the neutral position $\left(25.8 \pm 5.2 \mathrm{~cm} \mathrm{H}_{2} \mathrm{O}\right)$, oropharyngeal leak pressure was significantly higher with flexion $\left(28.5 \pm 3.4 \mathrm{~cm} \mathrm{H}_{2} \mathrm{O}, \mathrm{P}=0.015\right)$ and lower with extension $\left(23.0 \pm 4.2 \mathrm{~cm} \mathrm{H}_{2} \mathrm{O}, \mathrm{P}=0.015\right)$, but similar with rotation $\left(26.7 \pm 5.1 \mathrm{~cm} \mathrm{H}_{2} \mathrm{O}, \mathrm{P}=0.667\right)$. Flexion of 
the head and neck adversely affected the ventilation score compared with the neutral position $\mathrm{P}=0.004$. So they Concluded that effective ventilation with I-gel can be performed in patients in whom the head and neck is extended or rotated, whereas flexion of the head and neck adversely affects ventilation. Clinically, flexion of the head and neck should be avoided during ventilation with I-gel.

Mishra and Nawaz et al. [8] in their study compared the effect of head and neck position on the oropharyngeal leak pressures and fibreoptic view of the glottis and ventilation scores between ProSeal LMA and the I-gel. Compared with neutral position, oropharyngeal leak pressures were significantly higher with flexion and lower with extension but similar with rotation of head and neck. But the oropharyngeal leak pressure was significantly higher for ProSeal LMA compared with the I-gel in all positions. Peak airway pressures were significantly higher with flexion in both groups (however this did not affect ventilation), lower with extension in ProSeal group and comparable in I-gel group but did not change significantly with rotation of head and neck in both groups. They concluded that effective ventilation can be done with both ProSeal LMA and I-gel with head in all the above positions. ProSeal LMA has a better margin of safety than I-gel due to better sealing pressures except in flexion where the increase in airway pressure is more with the former. Extreme precaution should be taken in flexion position in ProSeal LMA.

In contrast to what Sanuki and Mishra concluded in their studies that I gel was not very efficient when used in the flexion position of the head, we demonstrated that using the Baska mask and the SLIPA ${ }^{\mathrm{TM}}$ when the head is flexed was effective.

Isserles and Rozenberg. [17] Suggested in their study, that neck flexion removes the longitudinal tension in the anterior pharyngeal muscles, allowing them to settle down onto the mask to form a better seal. Neck flexion causes a reduction in the anteroposterior diameter of the pharynx [18] This may explain the cause of a higher pressure seal in almost all the studies including our study when the head is flexed. The high sealing pressure and good ventilation score in different head position that the Baska mask has in comparison to other supraglottic devices may be due to its sealing mechanism that is flexible and more resilient then most of the sealing mechanisms of the other devices that enables the Baska mask to adapt to any change that occurs due to change in the position of the head and neck.

\section{Conclusions}

Based on the results of this study, effective ventilation is possible with both Baska mask and SLIPA ${ }^{\mathrm{TM}}$ in all head positions. As regard the air way sealing pressure, Baska mask has a better air way sealing pressure than SLIPA ${ }^{\mathrm{TM}}$, however, care should be taken with extreme flexion and the airway pressures need to be monitored carefully. Also we concluded that during fibreoptic evaluation of glottis, a lower score was obtained with flexion in both devices, but there was no affection on the ventilation, which was evident from adequate delivered tidal volumes and comparable levels of end-tidal $\mathrm{CO}_{2}$ between the neutral and flexion positions.

\section{References}

[1] Choi GJ, Kang H, Baek CW, Jung YH, Woo YC, Kim SH and Kim JG. Comparison of streamlined liner of the pharynx airway (SLIPA ${ }^{\mathrm{TM}}$ ) and laryngeal mask airway: a systematic review and meta-analysis. Anaesthesia 2015; 70: 613-22.

[2] Miller DM. \& Light D. Laboratory and clinical comparisons of the Streamlined Liner of the Pharynx Airway (SLIPA ${ }^{\mathrm{TM}}$ ) with the Laryngeal Mask Airway (LMA $\left.{ }^{\mathrm{TM}}\right)$. Anaesthesia 2003; 58(2): 136-42.

[3] Choi YM, Cha SM, Kang H et al. The clinical effectiveness of the streamlined liner of pharyngeal airway (SLIPA ${ }^{\mathrm{TM}}$ ) compared with the laryngeal mask airway Proseal ${ }^{\mathrm{TM}}$ during general anesthesia. Korean J Anesthesiol 2010; 58:450-57.

[4] Lange M, Smul TM, Zimmermann P, Roewer N, Kehl F. Comparison of Two Supraglottic Airway Devices: SLIPATM Versus LMATM. Abstracts of papers, Anesthesiology 2006; 105: Abstract A871; http://www.asaabstracts.com (Last accessed 22/11/2011)

[5] Alexiev V, Ochana A, Abdelrahman D, Coyne J, McDonnell JG, Toole DP, NeliganP. Comparison of the Baska mask with the single-use laryngeal mask airway in low-risk female patients undergoing ambulatory Surgery. Anaesthesia 2013; 68: 1026-32.

[6] Alexiev V, Salim A, Kevin LG, Laffey JG. An observational study of the Baska_ mask: a novel supraglottic airway. Anaesthesia 2012; 67: 640-5.

[7] Tom VZ, Stephen G. The Baska Mask $®$ —A new concept in Self-sealing membrane cuff extraglottic airway devices, using a sump and two gastric drains: A critical evaluation. Journal of Obstetric Anaesthesia and Critical Care 2012; 2 (1): 23-30.

[8] Mishra SK, Nawaz M, Satyapraksh MVS., Parida S, Bidkar PU, Hemavathy B, and Pankaj Kundra P. Influence of Head and Neck Position on Oropharyngeal Leak Pressure and Cuff Position with the ProSeal Laryngeal Mask Airway and the IGel: A Randomized Clinical Trial. Anesthesiol Res Pract 2015:1-7.

[9] Keller C, Brimacombe J, Keller K, Morris R. A comparison of four methods for assessing airway sealing pressure with the laryngeal mask airway in adult patients. Br J Anaesth 1999; 82: 286-7.

[10] Sanuki T, Uda R, Sugioka S, et al. The influence of head and neck position on ventilation with the i-gel airway in paralyzed, anaesthetized patients. Eur J Anaesthesiol 2011: 28(8): 59799.

[11] Brimacombe JR, Brimacombe JC, Berry AM, et al. A comparison of the laryngeal mask airway and cuffed oropharyngeal airway in anesthetized adult patients. Anesth. Analg 1998; 87(1):147-52.

[12] Somri M, Vaida S, Fornari G, Mendoza GR, et al. A randomized prospective controlled trial comparing the laryngeal tube suction disposable and the supreme laryngeal mask airway: the influence of head and neck position on oropharyngeal seal pressure. BMC Anesthesiology 2016; 16(87) DOI 10.1186/s12871-016-0237-7. 
[13] Anand LK, Goel N, Singh M, Kapoor D. Comparison of the Supreme and the ProSeal laryngeal mask airway in patients undergoing laparoscopic cholecystectomy: A randomized controlled trial. Acta Anaesthesiologica Taiwanica 2016; 54: 44-50.

[14] Keller C, Brimacombe J, and Puhringer F. A fibreoptic scoring system to assess the position of laryngeal mask airway devices. Interobserver variability and a comparison between the standard, flexible and intubating laryngeal mask airways. Anasthesiol Intensivmed Notfallmed Schmerzther 2000; 35(11): 692-94.

[15] Park SH, Han SH, Do SH, Kim JW, and Kim JH. The influence of head and neck position on the oropharyngeal leak pressure and cuff position of three supraglottic airway devices. Anesth Analg 2009; 108(1): 112-17.

[16] Xue FS, Mao P, Liu HP, et al. The effects of head flexion on airway seal, quality of ventilation and orogastric tube placement using the ProSealTM laryngeal mask airway. Anaesthesia 2008; 63: 979-85.

[17] Isserles SA. and Rozenberg B. LMA: Reduction of gas leak. Can J Anaesth 1995; 42(5): 449.

[18] Nandi PR, Charlesworth CH, Taylor SJ, Nunn JF, and Dore C J. Effect of general anaesthesia on the pharynx. Br J Anaesth 1991; 66(2): 157-62. 